\title{
Geniculate ganglion neurons have individual tastes
}

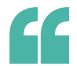

The majority of the imaged GGNs were singly tuned

5

Geniculate ganglion neurons (GGNs) receive inputs from taste receptor cells (TRCs) on the tongue, each of which selectively transduces one of five taste qualities: sweet, sour, salty, bitter and umami. However, the way in which taste-evoked signals from TRCs are processed by GGNs is unclear. In a recent study in Nature, Zuker and colleagues characterize the representation of different tastants by GGNs.

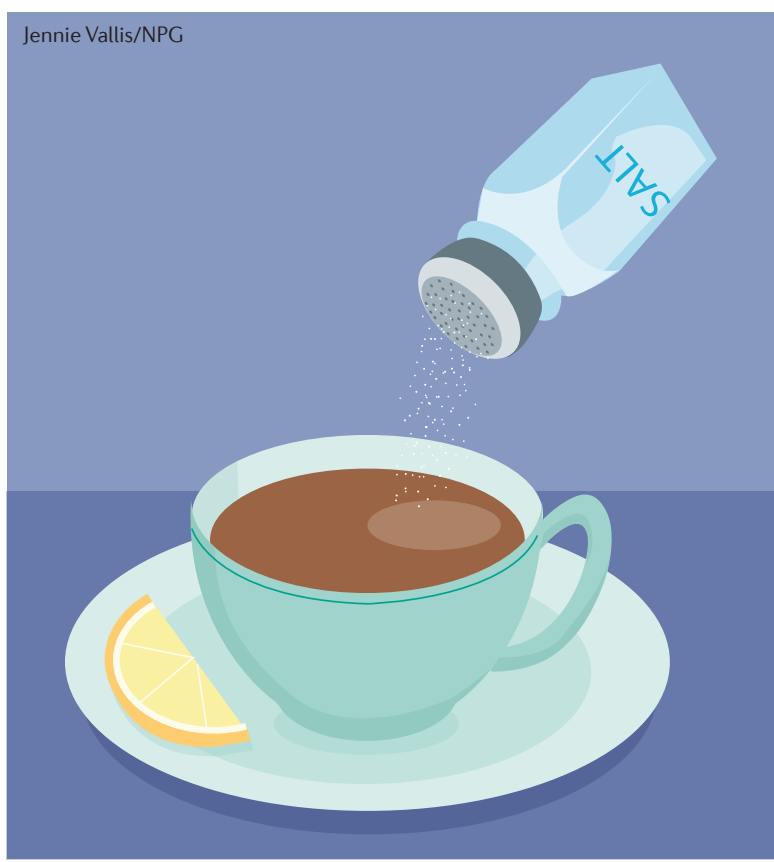

The authors generated mice that expressed the fluorescent calcium indicator GCaMP in GGNs genetically or virally, and imaged these neurons using two-photon microendoscopy. This technique enabled the fluorescent signal to be transmitted approximately $4 \mathrm{~mm}$ from the geniculate ganglion to the surface of the head. Tastant solutions - such as solutions of sucrose (sweet), sodium chloride (salty) or citric acid (sour) - were applied to the tongue, and the elicited GGN calcium responses were recorded from a total of 971 GGNs.

The majority of the imaged GGNs were singly tuned, in that they each responded to one of the five taste qualities. Moreover, GGNs that were sensitive to a particular taste could be silenced through genetic knockout of specific TRC receptors, or by application of selective receptor antagonists to the tongue. Solutions that contained two tastants induced calcium responses in the two ensembles of GGNs that had been activated by each tastant separately. Together, these findings indicate that most GGNs are singly tuned and respond specifically to a particular taste quality.

Of the GGNs that responded to more than one taste quality, most responded to bitter and sour tastants, which are normally detected by
TRCs that express taste receptor type 2 (T2R) family members and polycystic kidney disease 2-like 1 protein (PKD2L1), respectively. The authors sought to determine whether bitter-sour GGNs receive convergent inputs from T2R-expressing cells and PKD2L1-expressing cells, or single inputs from bitter-sensing cells that are sensitive to acid, independently of PKD2L1. Using a PKD2L1-targeted tetanus toxin, the authors ablated PKD2L1-expressing TRCs. There were no GGN calcium responses to sour alone in these mice; however, strikingly, there were still GGNs that showed a bitter-sour response, and this response was abolished by application of a T2R (bitter) antagonist onto the tongue. Therefore, the bitter-sour GGNs receive inputs from bitter TRCs that are sensitive to acid.

Through the use of this specialized imaging method, Zuker and colleagues show that taste-qualityspecific TRC signals are transmitted along 'labelled lines' to GGNs, which are thus tuned to a single taste quality.

Natasha Bray

ORIGINAL RESEARCH PAPER Barretto, R. P. J. et al. The neural representation of taste quality at the periphery. Nature http://dx.doi.org/10.1038/ nature13873 (2014) 